\title{
Coordinated Enhanced Surveillance with Healthcare Entities for Mass Gathering Events
}

\author{
Erin E. Austin* \\ Division of Surveillance and Investigation, Virginia Department of Health, Richmond, VA, USA
}

\section{Objective}

To describe the planning strategies and lessons learned by the Virginia Department of Health (VDH) when conducting enhanced surveillance during mass gathering events and coordinating with healthcare entities to distinguish event-related emergency department (ED) visits from community-related ED visits.

\section{Introduction}

Mass gatherings can result in morbidity and mortality from communicable and non-communicable diseases, injury, and bioterrorism. Therefore, it is important to identify event-related visits as opposed to community-related visits when conducting public health surveillance ${ }^{1}$. Previous mass gatherings in Virginia have demonstrated the importance of implementing enhanced surveillance to facilitate early detection of public health issues to allow for timely response ${ }^{2}$.

\section{Methods}

Between June 2015 and September 2015, VDH coordinated with two healthcare entities representing six acute care hospitals to conduct enhanced surveillance for the 2015 World Police and Fire Games and 2015 Union Cycliste Internationale (UCI) Road World Championships. VDH established initial communication with each healthcare entity between 1 week to 2 months before the event start date to discuss functional requirements with technical, informatics, and clinical staff. Requirements included: 1) health care entity identifying gathering attendees during the ED registration, 2) capturing a standardized mass gathering indicator within the patient's electronic health record (EHR), and 3) transmitting the gathering indicator to $\mathrm{VDH}$ through existing electronic syndromic surveillance reporting processes. ED visit records with the gathering indicator were analyzed by VDH using the Virginia Electronic Surveillance System for the Notification Community-based Epidemics (ESSENCE) and findings were incorporated in daily VDH situational reports. This same methodology will be applied for the upcoming U.S. Vice Presidential Debate in October 2016.

\section{Results}

The duration of the two gatherings in 2015 ranged from 9 to 10 days and the locations were categorized as urban. The population density of the gathering location ranged from 1,950 to 2,889 population per square mile. The estimated number of attendees ranged from 45,000 to 400,000 . Attendees were defined as having attended at least one day of the mass gathering event. The mass gathering indicator captured during the ED registration included the gathering acronym or a gathering specific field with a drop down menu containing true/false options. VDH utilized ESSENCE to identify 42 ED visits $(0.5 \%)$ with the gathering acronym out of 8,768 total ED visits during the 2015 World Police and Fire Games and 60 ED visits $(2.6 \%)$ with the gathering specific field out of 2,296 total visits during the 2015 UCI Road World Championships. The results of the U.S. Vice Presidential Debate in October 2016 are pending.

\section{Conclusions}

In 2015, VDH partnered with two healthcare entities to conduct enhanced surveillance during two mass gatherings. Although VDH routinely uses syndromic surveillance data to identify issues of public health concern, it has previously lacked the ability to identify ED visits specific to mass gatherings. Prior to collaboration with VDH, the healthcare entities did not capture gathering-specific ED visits using their EHR systems. The two healthcare entities successfully modified their business procedures and EHR system to capture and transmit a gathering indicator for ED visits despite some challenges. These challenges include constraints with customization of the EHR and syndromic surveillance systems, lack of standardized training among ED registration staff for interpreting and applying the gathering indicator, and limited functionality testing prior to the event. Lessons learned from this coordinated effort are to: 1) initiate the planning phase and identification of requirements as early as possible to ensure they are well defined and understandable, 2) implement frequent communications with the healthcare entity, and 3) customize requirements for the specific gathering as much as possible while balancing the burden and benefit to public health and the healthcare entity. The coordinated enhanced surveillance efforts provided both VDH and the healthcare entities with improved situational awareness and capacity building during mass gathering events. The strategies and lessons learned from these two events will be applied to improve enhanced surveillance of public health issues during future mass gatherings, including the U.S. Vice Presidential Debate in October 2016.

\section{Keywords}

Mass gatherings; Syndromic Surveillance; Healthcare

\section{References}

1. Steffen, Robert et al. Non-communicable health risks during mass gatherings. The Lancet Infectious Diseases. 2012;12 (2),142-149.

2. Centers for Disease Control and Prevention. "Surveillance for Early Detection of Disease Outbreaks at an Outdoor Mass GatheringVirginia, 2005," MMWR Morb. Mortal. Wkly. Rep. 2006; 55 (3),71-74. http://www.cdc.gov/mmwr/preview/mmwrhtml/mm5503a3.htm

\section{*Erin E. Austin}

E-mail: erin.austin@vdh.virginia.gov 